\title{
Using InGaN/GaN Multiple Quantum well Green light-Emitting Diodes as a Promising Replacement of Conventional light Sources
}

\author{
Shyam Sunder Manaktala, K.M.Singh
}

\begin{abstract}
In this particular paper we increase a graded indium composition p type InGaN (p InGaN) conduction level to supplant the p type AlGaN electron blocking level \& a p GaN level to update the mild yield intensity of a GaN based green light transmitting diode (LED). The indium structure of the $p$ InGaN coating reduced from $10.4 \%$ to zero \% across the development heading. A tale configuration is proposed for n-electrode with openings to be connected in Thin-GaN light-transmitting diodes (LEDs). The impact of the n-electrode with gaps on the thermal and electrical qualities of a Thin-GaN LED chip is researched utilizing a three-dimensional numerical simulation The IQE of green LED is restricted by the deformities and the internal electric field in $M Q W$. Thusly, we talk about the ongoing advancement in improving the IQE of green LED in detail. These techniques can be partitioned into two classes. A portion of these techniques were proposed to upgrade precious stone nature of InGaN/GaN MQW with high. In composition and low thickness of deformities by adjusting the development conditions. Different strategies concentrated on expanding electron-hole wave function cover by dispensing with the polarization impact.
\end{abstract}

Index Terms: electron, layer, electrodes, hole, density temperature, etc.

\section{INTRODUCTION}

GaN-based light emitting diodes (LEDs) have yanked for substantial concern and also have been considered a promising replacement for traditional gentle options with probably the most current few years [1]. The effectiveness of pink LEDs is high, along with pink LEDs were commercially employed in several fields, for instance, burning, show, light correspondence, backdrop illumination [2-3], etc. Nevertheless, the inner quantum effectiveness (IQE) of GaN based eco-friendly LEDs remains less than that of pink LEDs, which is referred to as the "Green Gap" [4]. It hinders the greenish LED to be attached with Red-Green-Blue (RGB) burning, apparent light communication, along with full shading displays. A considerable polarization area and bad crystal quality [5] would be the concept explanations behind the lower IQE of natural LEDs with an impressive indium composition. Honestly, the very poor hole injection likewise assumes a crucial job in the

Revised Manuscript Received on December 22, 2018.

Shamsunder Manataklata, Research Scholar, Dept. Of ECE , JECRC University, Jaipur, India

K M Singh, Professor, Dept. Of ECE, JECRC University, Jaipur, India poor quantum effectiveness of GaN based LEDs. Numerous analysts have suggested methods that are several to manage this problem determined by band engineering on the electron obstructing level (EBL). Kim et al. used a functioning layer accommodating power grid coordinated InAlN EBL to enhance the quantum efficiency of natural LEDs [6]. A graded superlattice $\mathrm{AlGaN} / \mathrm{GaN}$ embeddings level was suggested by J. Kang et al. to increase the effectiveness of hole injection as well as execution of natural LEDs [7].

An InAlGaN/GaN superlattice, an AlGaN/InGaN superlattice, along with a composition graded AlGaN EBL had been the same used to lower the possible screen of holes without hurting the electron constrainment. An as of late proposed technique to enhance the attributes of $\mathrm{p}$ type $\mathrm{GaN}$ is polarization doping [8]. It uses the inner polarization of the buildings as well as material composition evaluating to encourage absolutely free electrons or maybe holes [9]. At any rate, the improvement temperature of $\mathrm{AlGaN}$ is in each and every case very high in order to enhance the crystal quality. The higher indium foods InGaN/GaN different quantum effectively (MQW) of natural LEDs will be damaged amid the high temperature procedure [10]. Right now there aren't a lot of accounts about the p type coating building meant to boost the gap injection of GaN primarily based LEDs.

\section{MECHANISMS OF LOW IQE FOR A (GaN) BASED GREEN LED}

The InGaN wells' indium structure demands a top indium structure to complete a great deal of wavelength eco-friendly LED. The typically low quantum effectiveness of InGaN/GaN eco-friendly MQW with the higher indium structure could be ascribed to a number of factors. In the very first place, it is difficult to develop excellent InGaN/GaN MQWs with an impressive indium composition. The stress brought approximately by cross section crisscross among InGaN wells as well as $\mathrm{GaN}$ barriers might increment together with the raise of indium structure in the QWs. Indium grouping incited by a rebel strain is observed when the well thickness is littler than the basic thickness [11]. Be that as it may, if the well is thicker than the basic thickness, the maverick strain may cause the rebel strain-in- duced deformities, for example, point surrenders [12], debasements [13], V-defects [14]. 


\section{Using InGaN/GaN Multiple Quantum well Green light-Emitting Diodes as a Promising Replacement of Conventional light Sources}

These high-density strain-induced imperfections may go about as nonradiative recombination focuses within the InGaN/GaN MQWs and, so decline the IQE of environmentally friendly LED.

Furthermore, the development of indium composition also prompts the material inhomogeneity of the InGaN effectively layers due to the very poor miscibility among $\mathrm{GaN}$ as well as InN. Phase segregation causes challenges in accomplishing homogeneous InGaN amalgams, and even a debasement of the InGaN/GaN MQW dynamic locale [15]. Additionally, there's an internal electrical area of $\mathrm{InGaN} / \mathrm{GaN}$ different quantum wells of LED created about the c'plane sapphire. The electrostatic area prompts the spatial separating of electron'hole wave works and consequently cuts down on the radiative recombination fees [16]. Since the piezoelectric polarization relies upon strain, the reduction in recombination rate is bigger for longer emission wavele gths' green LED.

\section{APPROACHES TO ENHANCE THE EFFICIENCY OF GREEN LED}

Numerous endeavors have been extended to defeat the difficulties referenced previously. As of late, a few approaches have been reported to adequately improve IQE of InGaN/GaN MQW green LED. These methodologies can be separated into two classifications. The main sort of strategies is centered around enhancement of radiative recombination by improving crystal quality of MQW. These strategies smothered the indium segregation and reduce the thickness of imperfection by streamlining the development state of MQW and utilizing some new structure. Another sort of methods in- wrinkled the recombination rate by raising the cover of wave function of holes and electrons, including polarization restraining and vitality band engineering plan.

\section{IMPROVEMENT OF CRYSTAL QUALITY}

Ordinarily, the perfect advancement temperature of InGaN properly layers is substantially less than that of $\mathrm{GaN}$ barrier levels due to lower miscibility of $\mathrm{InN}$ found $\mathrm{GaN}$ [seventeen]. The traditional strategy is developing the entire MQW compelling district in a con' stant minimal temperature since it's difficult to modify the improvement temperature precisely and quickly. Thus, the crystal quality of $\mathrm{GaN}$ obstacles created at temperature that is low is very bad to have excellent optoelectronic qualities, and that is steadily significant in eco-friendly LED with good indium factor. S.J. Chan et al. enhanced the crystal quality as well as mild yield severeness of InGaN/GaN MQW greenish LED by raising a $\mathrm{GaN}$ barrier at temperature that is higher [18].

Amid the development of InGaN/GaN MQW, they initially inclined down the temperature and held up till it balanced out to grow an InGaN well layer. From that point onward, temperature was increase to a higher esteem and held up till it balanced out to grow a GaN barrier layer. The detail of the temperature sloping procedure is appeared in Fig. 1(a). Figure 1(b) demonstrates the light yield control as a function of injection current. The $20-\mathrm{mA}$ yield intensity of nitride-based green LEDs with high temperature GaN barriers expanded by $65 \%$ compared to that of the conventional InGaN/GaN green LEDs.

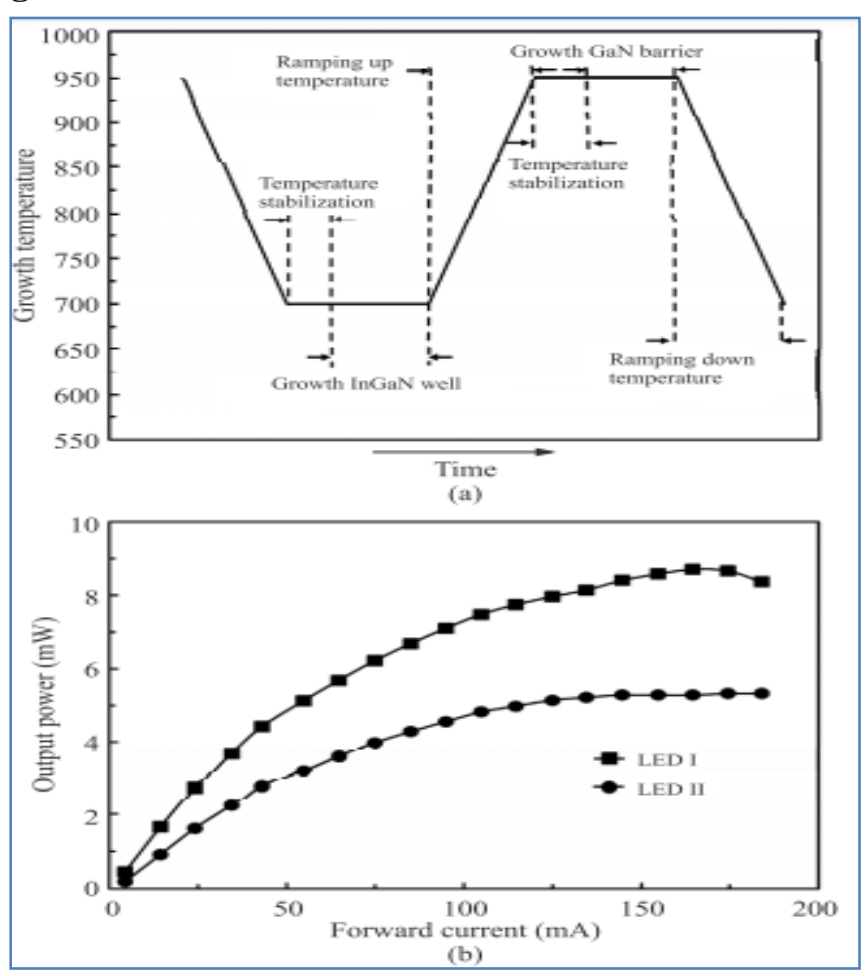

Figure 1: (a) Time sequence of the temperature ramping process during the growth of InGaN/GaN MQW active region.

(b) Output power of LEDI b (with a temperature ramping MQW) and LEDII (with a constant MQW).

Be that as it might, the high temperature advancement $\mathrm{GaN}$ barrier might possibly damage the InGaN effectively layer. Amid the temperature inclining as well as screen development process, the nicely experiences a greater temperature which prompts indium re-evaporation and segregation at the interface of QWs. This thermal debasement of MQW reduces the radiative recombination rate. Jin-Woo Ju et al. utilized a slight GaN, just as a protection layer to avoid thermal damage to the delicate well [19]. After the development of an InGaN well, a 1 nm-thick GaN well-protection-layer was along these lines covered at a similar temperature.

Michael Moseley's evaluation uncovered which indium area segregation was much more connected with the quantity of inside dium adsorbed on the counter, rather compared to improvement in the Inrich plan as a rule [20]. He proposed a method for managing indium area segregation by safeguarding Group III metal movements at a fixed harmony through metalmodulated epitaxy (MME). The rare turning off of the metallic emission cells requires into bank account complete utilization of overabundance metallic on the counter, retaining beads from continuing throughout the advancement.

HungCheng Lin et al. demonstrated the upsides of using indium therapy in the improvement of InGaN/GaN MQW [twenty one]. The indium therapy is usually to place in an extra progression where only NH3 and TMIn got the opportunity to stream directly into the reactor at each 
InGaNtoGaN interface amid the improvement of MQW. Subsequently, there's a sleek screen as well as minimal Vshape imperfection density in InGaN/GaN MQW, as appeared in Figure 2(a) as well as 2(b). Figure 2(c) shows the production energy of LED with as well as with no indium remedy. The light production energy of the TMIn addressed LED increments by forty three \% in contrast to which of untreated LED.

Yufeng $\mathrm{Li}$ et al. discovered that the GaN epilayer created on the created sapphire substrate demonstrated a forty four \% reduced threading dislocation density than which created on planar substrate [22]. A GaNbased earth-friendly LED created on $\mathrm{s}$ created cplane sapphire thus possessed a multiplying of the inner quantum efficiency.

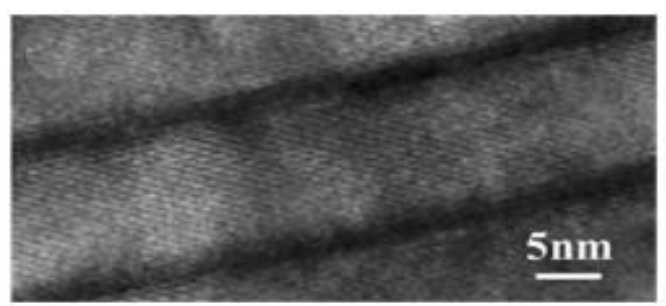

(a)

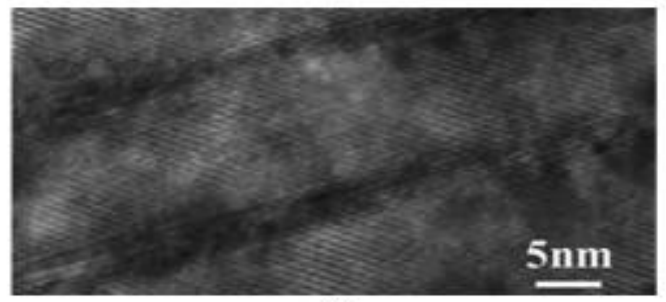

(b)

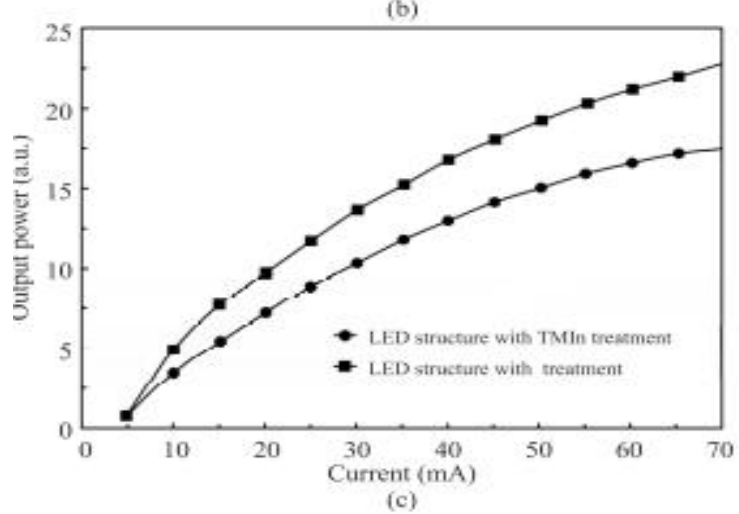

(c)

Figure 2: HRTEM images of the LEDs grown with (a) and without (b) indium treatment, and (c) light output-current characteristics.

Y. Yang et al. enhanced the crystallization quality as well as optoelectronic qualities of InGaN/GaN MQW of environmentally friendly LED by improving the components on freestanding GaN substrates [23]. The density of micro structural abandons within the LED on GaN was generously decreased. Appropriately, the IQE of environmentally friendly LED on GaN was seventy one \% above relative LED created on sapphire. The improvement american states on the MQW presume serious task within the crystallization qualities as well as gentle qualities of eco-friendly LED. In the meantime, the high'temperature post'growth on the p'GaN gap transportation level can make winter harm the InGaN/GaN MQW as well as reduces the emission advantages on the InGaN/GaN MQWs LED. The ther' mal harm on the $\mathrm{InGaN} / \mathrm{GaN} \mathrm{MQW}$, that is as a result of higher advancement heat of $\mathrm{p}^{\prime} \mathrm{GaN}$, is ascribed towards the indium dissemination into GaN screen levels coming from InGaN properly levels [24]. It is promising to bring down the improvement heat of a $\mathrm{p}^{\prime} \mathrm{GaN}$ level for more effective optical as well as fundamental qualities of InGaN/GaN MQW [25]. The caliber of InGaN/GaN MQW eco-friendly LED might similarly be enhanced by "active'region'friendly" p'InGaN levels created at heat that is lower .

\section{WAVE FUNCTIONS OVERLAP IMPROVEMENT OF ELECTRONS AND HOLES}

Polarization electric-powered field legitimately will cause the wave performs division of holes as well as electrons. The non polar as well as semi polar InGaN/GaN MQW might productively lessen the electrostatic area intensity as well as increment the quantum effectiveness of InGaN QW [26]. The rplane sapphire as well as aplane $\mathrm{SiC}$ have been used for the nonpolar aoriented nonpalor $\mathrm{GaN}$ advancement. Subsequently, aplane GaN platforms as well as mplane InGaN flicks on mplane 6HSiC had been accounted for [27] Semipolar InGaN/GaN eco-friendly LEDs had been also constructed on the mplane sapphire. Taeil Jung et al. found a semipolar eco-friendly InGaN/GaN MQW developed on great ease cplane sapphire substrates and achieved thirty \% increased inner quantum effectiveness when compared to a standard cplane MQW [28]. Additionally, bulkGaN substrates with very subjective introductions are attractive to develop a nonpolar as well as semipolar green LED, for instance, launch GaN semipolar GaN, and also aplane GaN [29]. In the study of TingWei Yeh et al, even GaN nanorod arrays have been created vertically by specific area advancement on cplane substrates. These nanorods exhibited 6 non polar 11 ? zero facets, that filled in as growth surfaces for semipoalr InGaN/GaN MQW [30], as came out in Figure 4(a) as well as 4(b). The nonappearance of polar airplane introduction prompts elimination or crucial decrease of polarization impacts.
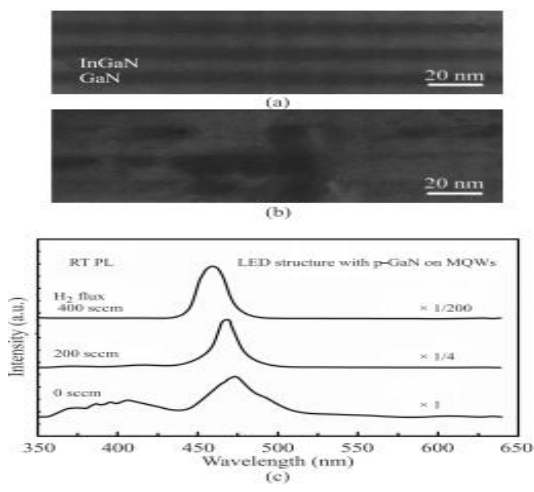

Figure 3. Cross sectional images of InGaN $(6 \mathrm{~nm}) / \mathrm{GaN}(8 \mathrm{~nm})$ MQWs grown (a) with $400 \mathrm{sccm} H 2$ and (b) without $\mathrm{H} 2$ during the interruption time of $0.5 \mathrm{~min}$., (c) the effect of $\mathrm{H} 2$

introduction on the RT PL of a blue LED structure. The $\mathrm{H} 2$ was introduced during the interruption time of 0.5 min. 


\section{Using InGaN/GaN Multiple Quantum well Green light-Emitting Diodes as a Promising Replacement of Conventional light Sources}

Assuaged QCSE was consequently achieved with a longer coverage of electronhole trend operates for MQW. Thusly, the chance of dipole alter within the semipolar as well as nonpolar QWs was improved for more and more effective radiative recombination [31]. Stress is straight within control of piezoelectric polarization. Decrease of stress is able to eliminate the piezoelectric area as well as enhance the covering of electronhole trend feature. There are several novel strategies suggested as of late to ease upwards the stress

A particular method that is powerful to lessen stress is a straincompensated advancement process that intends to cultivate MQW and have a novel framework to ease upwards stress. D.M. Van Den Broeck et al. disclosed the advancement of InxGa1xN/GaN "strainbalanced" many quantum wells (SBMQWs) produced on heavy InyGa1yN platforms for $\mathrm{x}>\mathrm{y}$ [32]. The SBMQW is a cross part synchronised on the heavy InyGa1yN structure. Within the SBMQWs, thicknesses \& compositions are selected with the aim which the compressive worry within the wells is modified through the flexible stress within the obstacles.

R. Arvind Pawan et al. planned a novel highlow indium make up and then prestrained the InGaN/GaN MQW system of environmentally friendly LED [33]. With this MQW the original $4 \mathrm{QWs}$ possess a reduced indium aspect contained InxGa1? $\mathrm{xN}$ to mini mize cross part stress and also achieve much better present density. Be that here as it might, the 5 th level has a greater indium part to obtain emission within the eco-friendly location of the entire spectrum. Such a scheme using an amount contained indium make up within the QWs impressively cuts down on the stresses to come down with powerful outcomes and place within the enhancement of outstanding severeness as well as radiative recombination fee.

Hongping Zhao et al. found a straincompen satiated InGaN/AlGaN QW system making use of fragile tensile strained $\mathrm{AlGaN}$ obstacles to cover the compressively emphasized InGaN QWs [34]. Additionally, Seoung Hwa $n$ Park et al. detailed a straincompensated InGaN/InGaN QW which has a tensilestrained InGaN screen [35]. (a)

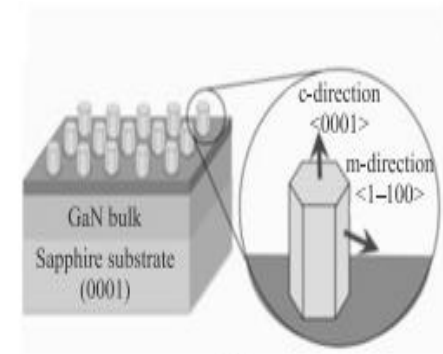

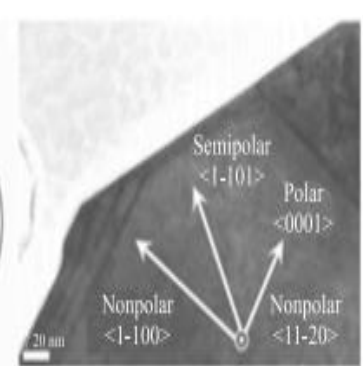

(b)

\section{Figure 4: (a) Schematic diagram of a GaN nanorod array, (b) InGaN/GaN MQWs are grown on three different planes as indicated in the arrows (reprinted from Ref. 36).}

The radiative efficiency of a strain-compensated QW structure is appeared to be a lot bigger than that of a conventional QW structure. This is ascribed to the reduction of internal field in the strain-compensated QW structure

\section{CONCLUSION}

Overall, we've examined the device restricted the inner quantum effectiveness of InGaN/GaN MQW greenish LED and late techniques In order to improve the IQE. To accomplish greener emission, loaded with composition in InGaN QW is needed. So, a sizable cross section jumble among InGaN wells as well as GaN barriers occurs as the In composition

in wrinkles. This effect leads straininduced abandons and sound polarization field. The flaws go approximately as nonradiative recombinative concentrates \& polarization isolates the electronhole trend feature. Subsequently, the radiative recombination effectiveness of InGaN/GaN MQW eco-friendly LED is less than that of green LED that is categorized "green gap". This way, we've place accentuation on the continuing endeavors made to improve effectiveness of natural LED. A portion of these techniques were pro presented to build good quality InGaN/GaN MQW with higher. In composition and decreased density of imperfections by altering the development conditions. Some other techniques concentrated on wiping away the polarization effect in MQW by nonpolar and semipolar LED or maybe novel MQW structures.

\section{REFERENCES}

1. Wang, L.; Liu, Z.; Zhang, Z.H.; Tian, Y.D.; Yi, X.; Wang, J.; Li, J.; Wang, G. Interface and photoluminescence characteristics of graphene-(GaN/InGaN)n multiple quantum wells hybrid structure. J. Appl. Phys. 2016, 119, 611-622. [CrossRef]

2. Wang, Q.; Ji, Z.; Zhou, Y.; Wang, X.; Liu, B.; Xu, X.; Gao, X.; Leng, J. Diameter-dependent photoluminescence properties of strong phase-separated dual-wavelength InGaN/GaN nanopillar LEDs. Appl. Surf. Sci. 2017, 410, 196-200. [CrossRef]

3. Pimputkar, S.; Speck, J.S.; Denbaars, S.P.; Nakamura, S. Prospects for LED lighting. Nat. Photonics 2009, 3, 180-182. [CrossRef]

4. Anandan, M. Progress of LED backlights for LCDS. J. Soc. Inf. Displ. 2008, 16, 287-310. [CrossRef]

5. Soon, C.M. White Light Emitting Diode as Liquid Crystal Display Backlight; Massachusetts Institute of Technology: Cambridge, MA, USA, 2007

6. Zhou, Q.; Xu, M.; Wang, H. Internal quantum efficiency improvement of InGaN/GaN multiple quantum well green light-emitting diodes. Opto-Electron. Rev. 2016, 24, 1-9. [CrossRef]

7. Ren, P.; Zhang, N.; Xue, B.; Liu, Z.; Wang, J.; Li, J. A novel usage of hydrogen treatment to improve the indium incorporation and internal quantum efficiency of green InGaN/GaN multiple quantum wells simultaneously. J. Phys. D Appl. Phys. 2016, 49, 175101. [CrossRef]

8. Kim, H.J.; Choi, S.; Kim, S.-S.; Ryou, J.-H.; Yoder, P.D.; Dupuis, R.D.; Fischer, A.M.; Sun, K.; Ponce, F.A. Improvement of quantum efficiency by employing active-layer-friendly lattice-matched InAlN electron blocking layer in green light-emitting diodes. Appl. Phys. Lett 2010, 96, 101102. [CrossRef] Nanomaterials 2018, 8, 5127 of 8

9. Kang, J.; Li, H.; Li, Z.; Liu, Z.; Ma, P.; Yi, X.; Wang, G. Enhancing the performance of green $\mathrm{GaN}$-based light-emitting diodes with graded superlattice AlGaN/GaN inserting layer. Appl. Phys. Lett. 2013, 103 , 102104. [CrossRef]

10. Kivisaari, P.; Oksanen, J.; Tulkki, J. Polarization doping and the efficiency of III-nitride optoelectronic devices. Appl. Phys. Lett. 2013, 103, 1029. [CrossRef]

11. Ju, J.W.; Zhu, J.; Kim, H.S.; Lee, C.R.; Lee, I.H. Effects of p-GaN growth temperature on a green InGaN/GaN multiple quantum well. J Korean Phys. Soc. 2007, 50, 810. [CrossRef]

12. Y.S. Lin, K.J. Ma, C. Hsu, S. W. Feng, Y.C. Cheng, C.C. Liao, C.C. Yang, C.C. Chou, C.M. Lee, and J.I. Chyi, "De- pendence of composition fluctuation on indium content in InGaN/GaN multiple quantum wells", Appl. Phys. Lett. 77, 2988-2990 (2000). 
13. J.S. Yue, O. Brandt, and K.H. Ploog, "Photoluminescence intensity of GaN films with widely varying dislocation den- sity”, J. Mater. Res. $18,1247-250$ (2003)

14. D.D. Koleske, A.E. Wickenden, R.L. Henry, and M.E. Twigg, "Influence of MOVPE growth conditions on carbon and silicon concentrations in GaN", J. Cryst. Growth 242, 55-69 (2002).

15. C.J. Sun, M.Z. Anwar, Q. Chen, J.W. Yang, M.A. Khan, M.S. Shur, A.D. Bykhovski, Z. Liliental-Weber, C. Kisie- lowski, M. Smith, J.Y. Lin, and H.X. Jiang, "Quantum shift of band-edge stimulated emission in InGaN-GaN multiple quantum well light-emitting diodes (vol 70, p. 2978, 1997)", Appl. Phys. Lett. 98, 2978-2980 (1997)

16. F. Scholz, A. Sohmer, J. Off, V. Syganow, A. Dornen, J.S. Im, A. Hangleiter, and H. Lakner, "In incorporation effi- ciency and composition fluctuations in MOVPE grown GaInN/GaN hetero structures and quantum wells", Materials Science And Engineering B-Solid State Materials For Ad- vanced Technology 50, 238-244 (1997).

17. M.D. McCluskey, L.T. Romano, B.S. Krusor, N.M. Johnson, T. Suski, and J. Jun, "Interdiffusion of In and Ga in InGaN quantum wells", Appl. Phys. Lett. 73, 1281-1283 (1998)

18. R. Jae-Hyun, P.D. Yoder, L. Jianping, Z. Lochner, K. Hyu- nsoo, C. Suk, J.K. Hee, and R.D. Dupuis, "Control of quantum-confined stark effect in InGaN-based quantum wells", IEEE J. Sel. Top. Quant. 15, 1080-1091 (2009)

19. H. I-Hsiu and G.B. Stringfellow, "Solid phase immiscibility in GaInN", Appl. Phys. Lett. 69, 2701-2703 (1996).

20. C.H. Liu, Y.K. Su, T.C. Wen, S.J. Chang, and R.W. Chuang, "Nitride-based green light emitting diodes grown by temper- ature ramping”, J. Cryst. Growth. 254, 336-341 (2003).

21. L.W. Wu, S.J. Chang, Y.K. Su, R.W. Chuang, T.C. Wen, C.H. Kuo, W.C. Lai, C.S. Chang, J.M. Tsai, and J.K. Sheu, "Nitride-based green light-emitting diodes with high temper- ature GaN barrier layers", IEEE T. Electron. Dev. 50, 1766-1770 (2003).

22. J. Ju, H. Kim, L. Jang, J.H. Baek, D. Shin, and I. Lee, “A well protection layer as a novel pathway to increase indium com- position: a route towards green emission from a blue InGaN/ GaN multiple quantum well", Nanotechnology 18, 460-464 (2007).

23. M. Moseley, J. Lowder, D. Billingsley, and W.A. Doolittle, "Control of surface adatom kinetics for the growth of high- -indium conten InGaN throughout the miscibility gap", Appl. Phys. Lett. 97, 191902-191902-3 (2010).

24. L. Hung-Cheng, L. Ruo-Syuan, and C. Jen-Inn, "Enhancing the quantum efficiency of InGaN green light-emitting diodes by trimethylindium treatment”, Appl. Phys. Lett. 92, 161111-161113 (2008).

25. L. Yufeng, Y. Shi, Z. Mingwei, Z. Liang, H. Wenting, T. Detchprohm, Y. Taniguchi, N. Tamura, S. Tanaka, and C. Wetzel, "Defect-reduced green GaInN/GaN light-emitting diode on nanopatterned sapphire", Appl. Phys. Lett. 98, 151102-151103 (2011).

26. Y. Yang, X.A. Cao, and C.H. Yan, "Rapid efficiency roll-off in high-quality green light-emitting diodes on freestanding GaN substrates", Appl. Phys. Lett. 94, 041117-041117-3 (2009).

27. M. Oh, M. Kwon, I. Park, S. Baek, S. Park, S.H. Lee, and J.J. Jung, "Improvement of green LED by growing $\mathrm{p}-\mathrm{GaN}$ on $\mathrm{In} 0.25 \mathrm{GaN} / \mathrm{GaN}$ MQWs at low temperature", J. Cryst. Growth. 289, 107112 (2006).

28. W. Lee, J. Limb, J.H. Ryou, D. Yoo, T. Chung, and R.D. Dupuis, "Influence of growth temperature and growth rate of $\mathrm{p}-\mathrm{GaN}$ layers on the characteristics of green light emitting diodes", J. Electron. Mater. 35, 587-591 (2006).

29. S. Qian and H. Jung, "Nonpolar and semipolar GaN he- teroepitaxy on sapphire for LED application”, Proc. SPIE 7617, 761714-761717 (2010)

30. M. Iwaya, A. Miura, R. Senda, T. Nagai, T. Kawashima, D. Iida, S. Kamiyama, H. Amano, and I. Akasaki, "Control of stress and crystalline quality in GaInN films used for green emitters", J. Cryst. Growth 310, 4920-4922 (2008).

31. T. Jung, T. Jung, L. Lee, L. Lee, P.C. Ku, and P.E. Ku, "Semi-polar green LEDs on c-plane sapphire substrates", IEEE, 571-572, (2008).

32. T. Detchprohm, M. Zhu, Y. Li, Y. Xia, C. Wetzel, E.A. Preble, L. Liu, T. Paskova, and D. Hanser, "Green light emit- ting diodes on a-plane GaN bulk substrates", Appl. Phys. Lett. 92, 241109-241109-3 (2008).

33. G. You, J. Liu, Z. Jiang, L. Wang, N.A. El-Masry, A.M. Hosalli, S.M Bedair, and J. $\mathrm{Xu}$, "Enhanced radiative recom- bination and suppressed Auger process in semipolar and nonpolar InGaN/GaN quantum wells grown over GaN na- nowires", Opt. Lett. 39, 1501-1504 (2014)

34. D.M. Van den Broeck, D. Bharrat, A.M. Hosalli, N.A. El--Masry, and S.M. Bedair, "Strain-balanced InGaN/GaN mul tiple quantum wells", Appl. Phys. Lett. 105, 031107-031107-5 (2014).
35. P.R. Arvind, P.R. Arvind, C. Dhanavantri, and C. Dhanavan- tri, "Optimization of optical behavior of InGaN-GaN MQW green LEDs with a novel high-low profile of Indium compo- sition in the active layer", IEEE, pp. 748-751 (2009).

36. H. Zhao, R.A. Arif, Y. Ee, and N. Tansu, "Self-consistent analysis of strain-compensated InGaN-AlGaN quantum wells for lasers and light-emitting diodes”, IEEE J Quantum Elect 45, 66-78 (2009).

\section{Authors Profile}

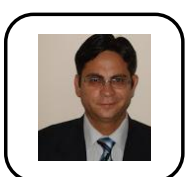

Shyam Sunder Manaktala is an Assistant Professor, in the Department of Electronics and Communication Engineering , Jaipur Engineering College and Research Centre, jaipur. He received B.E. degree from Department of ECE, Nagpur University, in 1997. He did PGDASDD (post graduate diploma) from ER\&DCI Noida by in 2000.He obtained M.TECH.(VLSI DESIGN) degree from MNIT, Jaipur in 2010. Presently he is pursuing PhD. Under ECE department from JECRC, University Jaipur on "Performance Enhancement of LED using Nanomaterials". He has more than 19 years of teaching and research experience in ECE and Information Technology. He has served various administrative assignments in different organizations. He has authored 3 books under university curriculum. His 7 research/review papers have been published in various Indian and International Journals of repute. He is presently member of several international professional organizations like IAENG,WASET,ACADEMIA,RESEARCHGATE. He is the author of blog on career articles for ECE engineers, for fresh ECE engineering graduates. $\mathrm{He}$ is the recipient of various awards and honors in the field of Education as best faculty mentor award, scholarship by HRD, SCJP, IBM DB2 Associate. His area of research include Nanotechnology, Optical communication, VLSI Design

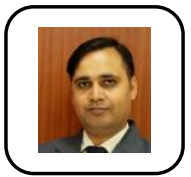

Dr. K.M.Singh is a Professor, in the Department of Electronics and Communication Engineering, JECRC, University, jaipur. He did B.E. in Electronics Engg from HBTI, Kanpur in 1999. He received Ph.D. in Nanotechnology from Jadavpur University, Kolkata in 2011.He has 20 years of teaching and research experience. He has served in several reputed organizations in different states in India for various administrative, educational teaching and research assignments. $\mathrm{He}$ is life time member of professional organization ISTE . His area of research include Nanotechnology, image and video processing, signal processing, solar cell and embedded system. His 08 research/review papers have been published in conferences and 18 papers are published in various Indian and International Journals of repute. 